\title{
CRITERIA FOR THE BOUNDEDNESS AND COMPACTNESS OF INTEGRAL TRANSFORMS WITH POSITIVE KERNELS
}

\section{A. MESKHI}

A. Razmadze Mathematical Institute, Georgian Academy of Sciences, 1 M. Aleksidze St., Tbilisi 380093, Georgia (meskhi@rmi.acnet.ge)

(Received 30 June 1999)

\footnotetext{
Abstract The necessary and sufficient conditions that guarantee the boundedness and compactness of integral operators with positive kernels from $L^{p}(a, b)$ to $L_{\nu}^{q}(a, b)$, where $p, q \in(1, \infty)$ or $0<q \leqslant 1<p<$ $\infty$, for a non-negative Borel measure $\nu$ on $(a, b)$ are found.

Keywords: boundedness; compactness; weight; operators with positive kernels; measure of non-compactness

AMS 2000 Mathematics subject classification: Primary 46B50; 47B34; 47B38
}

\section{Introduction}

In the present work we find the necessary and sufficient conditions for the boundedness and compactness of the operator

$$
K(f)(x)=\int_{a}^{x} k(x, y) f(y) \mathrm{d} y
$$

from $L^{p}(a, b)$ to $L_{\nu}^{q}(a, b)(p, q \in(1, \infty)$ or $0<q \leqslant 1<p<\infty,-\infty<a<b \leqslant \infty$ and $\nu$ is a non-negative $\sigma$-finite Borel measure on $(a, b))$.

Analogous problems for the Riemann-Liouville type operator

$$
R_{\alpha} f(x)=\int_{0}^{x} \frac{f(y)}{(x-y)^{1-\alpha}} \mathrm{d} y,
$$

with $a=0, b=+\infty, p, q \in(1, \infty)$ and $\alpha>1 / p$ are solved in $[\mathbf{1 3}, \mathbf{1 4}]$ (for the case where $p=q=2$ and $\nu$ is absolute continuous see [15]). For the boundedness and compactness criteria of operators with power-logarithmic kernels

$$
I_{\alpha, \beta}(f)(y)=\int_{0}^{x}(x-y)^{\alpha-1} \ln ^{\beta}\left(\frac{\gamma}{x-y}\right) f(y) \mathrm{d} y
$$

with $0<b \leqslant \gamma<\infty, \alpha>1 / p$ and $\beta \geqslant 0$ see [10]. 
A complete description of the weight pairs $(v, w)$, which guarantee the boundedness of the operators with positive kernels from $L_{w}^{p}$ to $L_{v}^{q}$ when $1<p<q<\infty$, is given in [6] (see also [7, Chapter 3]).

Two-weight criteria for the boundedness of the operator $R_{\alpha}$ from $L_{w}^{p}(0, \infty)$ to $L_{v}^{q}(0, \infty)$ for $\alpha>1$ were found in [11] for $1<p \leqslant q<\infty$ and in [19] for $1<p, q<\infty$. An analogous problem for the Hardy operator,

$$
H f(x)=\int_{0}^{x} f(t) \mathrm{d} t
$$

was solved in $[\mathbf{2}, \mathbf{9}, \mathbf{1 2}]$ for $1<p \leqslant q<\infty$, and in [12] for $1<q<p<\infty$.

In the non-compact case we give the upper and the lower bound for the distance of $K$ from the subspace of compact operators from $L^{p}(a, b)$ to $L_{v}^{q}(a, b)$ when $1<p \leqslant q<\infty$.

\section{Preliminaries}

Let $\nu$ be a non-negative $\sigma$-finite Borel measure on $(a, b)$. Denote by $L_{\nu}^{q}(a, b)(0<q<\infty)$ a class of all $\nu$-measurable functions $g:(a, b) \rightarrow \mathbb{R}^{1}$ for which

$$
\|g\|_{L_{\nu}^{q}(a, b)}=\left(\int_{(a, b)}|g(x)|^{q} \mathrm{~d} \nu\right)^{1 / q}<\infty .
$$

If $\nu$ is absolutely continuous (i.e. $\mathrm{d} \nu=v(x) \mathrm{d} x$, where $v$ is a positive Lebesgue-measurable function on $(a, b))$, then the symbol $L_{v}^{q}(a, b)$ is used instead of $L_{\nu}^{q}(a, b)$. If $\nu$ is the Lebesgue measure, then we shall use the symbol $L^{q}(a, b)$.

The following lemma is known for the case $a=0$ and $b=\infty$ (see $[\mathbf{1 2}, \S 1.3]$ ), but we give the proof in the case where $-\infty<a<b \leqslant+\infty$ for completeness.

Lemma 2.1. Let $-\infty<a<b \leqslant+\infty, 1<p \leqslant q<\infty$ and let $\mu$ be a non-negative Borel measure on $(a, b)$. The inequality

$$
\left(\int_{(a, b)}\left|\int_{a}^{x} f(y) \mathrm{d} y\right|^{q} \mathrm{~d} \mu\right)^{1 / q} \leqslant c\left(\int_{a}^{b}|f(y)|^{p} \mathrm{~d} y\right)^{1 / p},
$$

where the positive constant $c$ does not depend on $f$, holds if and only if

$$
A=\sup _{a<t<b}(\mu([t, b)))^{1 / q}(t-a)^{1 / p^{\prime}}<\infty,
$$

where $p^{\prime}=p /(p-1)$. Moreover, if $c$ is the best constant in (2.1), then $A \leqslant c \leqslant 4 A$.

Proof. Let $f \geqslant 0, f \in L^{p}(a, b)$ and let

$$
\int_{a}^{b} f(y) \mathrm{d} y \in\left(2^{m}, 2^{m+1}\right]
$$

for some integer $m$. Denote

$$
\int_{a}^{x} f(y) \mathrm{d} y \equiv I(x)
$$


then for every $x \in(a, b)$ we have $I(x) \leqslant\|f\|_{L^{p}(a, b)}(x-a)^{1 / p^{\prime}}<\infty$. The function $I$ is continuous on $(a, b)$. Therefore, for every $k \in \mathbb{Z}$, with $k \leqslant m$, there exists $t_{k}$ such that $2^{k}=I\left(t_{k}\right)=\int_{t_{k}}^{t_{k+1}} f(y) \mathrm{d} y$ for $k \leqslant m-1$ and $2^{m}=I\left(t_{m}\right)$.

It is easy to verify that the sequence $\left\{t_{k}\right\}$ is increasing. Let $\alpha=\lim _{k \rightarrow-\infty} t_{k}$. Then we have $(a, b)=(a, \alpha] \cup\left(\cup_{k \leqslant m} E_{k}\right)$, where $E_{k}=\left[t_{k}, t_{k+1}\right)$ and $t_{m+1}=b$. When

$$
\int_{a}^{b} f(y) \mathrm{d} y=\infty
$$

we have $(a, b)=(a, \alpha] \cup\left(\cup_{k=-\infty}^{+\infty} E_{k}\right)$ (i.e. $\left.m=+\infty\right)$. If $t \in(a, \alpha)$, then $I(t)=0$ and if $t \in E_{k}$, then $I(t) \leqslant I\left(t_{k+1}\right) \leqslant 2^{k+1}$.

We have

$$
\begin{aligned}
\left(\int_{(a, b)}\left(\int_{a}^{x} f(y) \mathrm{d} y\right)^{q} \mathrm{~d} \mu\right)^{p / q} & \left(\sum_{k \leqslant m} \int_{E_{k}}(I(x))^{q} \mathrm{~d} \mu\right)^{p / q} \\
& \leqslant \sum_{k \leqslant m}\left(\int_{E_{k}}(I(x))^{q} \mathrm{~d} \mu\right)^{p / q} \leqslant \sum_{k \leqslant m} 2^{(k+1) p}\left(\int_{E_{k}} \mathrm{~d} \mu\right)^{p / q} \\
& =4^{p} \sum_{k \leqslant m} 2^{(k-1) p}\left(\mu\left(E_{k}\right)\right)^{p / q}=4^{p} \sum_{k \leqslant m}\left(\int_{t_{k-1}}^{t_{k}} f(y) \mathrm{d} y\right)^{p}\left(\mu\left(E_{k}\right)\right)^{p / q} \\
& \leqslant 4^{p} \sum_{k \leqslant m}\left(\int_{t_{k-1}}^{t_{k}}(f(y))^{p} \mathrm{~d} y\right)\left(t_{k}-t_{k-1}\right)^{p-1}\left(\mu\left(E_{k}\right)\right)^{p / q} \\
& \leqslant 4^{p} A^{p}\|f\|_{L^{p}(a, b)}^{p} .
\end{aligned}
$$

To prove the necessity, we put $f(y)=\chi_{(a, t)}(y)$ in $(2.1)$, where $t \in(a, b)$. Then we have $\|f\|_{L^{p}(a, b)}=(t-a)^{1 / p}$. On the other hand,

$$
\left(\int_{(a, b)}\left(\int_{a}^{x} f(y) \mathrm{d} y\right)^{q} \mathrm{~d} \mu\right)^{1 / q} \geqslant(\mu([t, b)))^{1 / q}(t-a),
$$

and consequently we obtain $A \leqslant c$.

We also need the following lemma.

Lemma 2.2. Let $-\infty<a<b \leqslant+\infty, 0<q<p<\infty$ and let $p>1$. Then the inequality

$$
\left(\int_{a}^{b}\left|\int_{a}^{x} f(y) \mathrm{d} y\right|^{q} v(x) \mathrm{d} x\right)^{1 / q} \leqslant c\left(\int_{a}^{b}|f(y)|^{p} \mathrm{~d} y\right)^{1 / p},
$$

where the positive constant $c$ does not depend on $f$, is fulfilled if and only if

$$
\bar{A}=\left(\int_{a}^{b}\left(\int_{x}^{b} v(t) \mathrm{d} t\right)^{p /(p-q)}(x-a)^{p(q-1) /(p-q)} \mathrm{d} x\right)^{(p-q) / p q}<\infty .
$$


Moreover, there exist positive constants $c_{1}$ and $c_{2}$ depending only on $p$ and $q$ such that if $c$ is the best constant in (2.2), then

$$
c_{1} \bar{A} \leqslant c \leqslant c_{2} \bar{A}
$$

This lemma can be proved in the same way as Lemma 1.3.2 of [12] (for the case $0<q<1<p<\infty$, see, for example, [18]).

We also need the following theorem, which can be obtained, for example, from Lemma 2 in Chapter XI of $[\mathbf{8}]$.

Theorem A. Let $1<p, q<\infty$ and let $-\infty<a<b \leqslant+\infty$. Suppose that $T$ : $L^{p}(a, b) \rightarrow L_{\nu}^{q}(a, b)$ is an integral operator of the type $T f(x)=\int_{a}^{b} T_{1}(x, y) f(y) \mathrm{d} y$, where $\nu$ is a $\sigma$-finite, separable measure on $(a, b)$ (i.e. $L_{\nu}^{q}(a, b)$ is separable). If

$$
\bar{A}=\|\| T_{1}(x, \cdot)\left\|_{L^{p^{\prime}(a, b)}}\right\|_{L_{\nu}^{q}(a, b)}<\infty,
$$

then the operator $T$ is compact from $L^{p}(a, b)$ to $L_{\nu}^{q}(a, b)$.

Definition 2.3. Let $-\infty<a<b \leqslant+\infty$. A kernel $k:\{(x, y): a<y<x<b\} \rightarrow$ $(0, \infty)$ belongs to $V(k \in V)$ if there exists a positive constant $d_{1}$ such that for all $x, y, z$ with $a<y<z<x<b$ the inequality

$$
k(x, y) \leqslant d_{1} k(x, z)
$$

holds.

Definition 2.4. Let $-\infty<a<b \leqslant+\infty$. We say that $k$ belongs to $V_{\lambda}\left(k \in V_{\lambda}\right)$ $(1<\lambda<\infty)$ if there exists a positive constant $d_{2}$ such that for all $x, x \in(a, b)$ the inequality

$$
\int_{a+(x-a) / 2}^{x} k^{\lambda^{\prime}}(x, y) \mathrm{d} y \leqslant d_{2}(x-a) k^{\lambda^{\prime}}(x, a+(x-a) / 2),
$$

is fulfilled, where $\lambda^{\prime}=\lambda /(\lambda-1)$.

Let $k_{1}$ be a positive measurable function on $(0, b-a)$ (if $b=\infty$, then we assume that $b-a=\infty)$.

Definition 2.5. Let $-\infty<a<b \leqslant+\infty$. We say that $k_{1}$ belongs to $V_{1 \lambda}\left(k_{1} \in V_{1 \lambda}\right)$ $(1<\lambda<\infty)$ if there exists a positive constant $d_{3}$ such that the inequality

$$
\int_{0}^{(x-a) / 2} k_{1}^{\lambda^{\prime}}(y) \mathrm{d} y \leqslant d_{3}(x-a) k_{1}^{\lambda^{\prime}}((x-a) / 2), \quad \lambda^{\prime}=\lambda /(\lambda-1),
$$

is fulfilled for all $x, x \in(a, b)$.

It is easy to verify that if $k_{1}$ is a non-increasing function on $(0, b-a)$ and $k_{1} \in V_{1 \lambda}$, then the kernel $k(x, y) \equiv k_{1}(x-y)$ belongs to $V \cap V_{\lambda}$.

Now we give some examples of kernels satisfying the above-mentioned conditions. 
Let $-\infty<a<b \leqslant+\infty$ and let $k(y)=y^{\alpha-1}$, where $\alpha>0$. If $1<\lambda<\infty$ and $1 / \lambda<\alpha \leqslant 1$, then $k_{1} \in V_{1 \lambda}$, and, consequently, the kernel $k(x, y) \equiv k_{1}(x-y)$ belongs to $V \cap V_{\lambda}$.

Assume that $-\infty<a<b<+\infty, b-a \leqslant \gamma<\infty, 1 / \lambda<\alpha \leqslant 1$ and $\beta \geqslant 0$. Let $k_{1}(y)=y^{\alpha-1} \ln ^{\beta}(\gamma / y)$. Then $k_{1} \in V_{1 \lambda}$ and, therefore, $k(x, y) \equiv k_{1}(x-y)$ belongs to $V \cap V_{\lambda}$.

Now suppose that $-\infty<a<b \leqslant+\infty$,

$$
k(x, y)=(x-y)^{\alpha-1} \ln ^{\beta-1}\left(\frac{x-a}{y-a}\right),
$$

where $1 / \lambda<\alpha \leqslant 1$ and $1-\alpha+1 / \lambda<\beta \leqslant 1$. Then $k \in V \cap V_{\lambda}$.

Let $a=0,0<b \leqslant+\infty$ and let $k(x, y)=x^{-\sigma(\alpha+\eta)}\left(x^{\sigma}-y^{\sigma}\right)^{\alpha-1} y^{\sigma \eta+\sigma-1}$ be the Erdelyi-Kober kernel, where $\sigma>0$ and $0<\alpha \leqslant 1$. It easy to see that if $1 / \lambda<\alpha \leqslant 1$ and $\eta>1 / \sigma-1$, then $k \in V \cap V_{\lambda}$.

Some results about integral transforms with the above-mentioned kernels can be found in $[\mathbf{1 7}]$.

\section{The boundedness criteria}

In this section we find the boundedness criteria for the integral operators with positive kernels.

Theorem 3.1. Let $-\infty<a<b \leqslant+\infty$. Suppose that $1<p \leqslant q<\infty$ and $k \in V \cap V_{p}$. Then the operator $K$ is bounded from $L^{p}(a, b)$ to $L_{\nu}^{q}(a, b)$ if and only if

$$
B \equiv \sup _{a<t<b}\left(\int_{[t, b)} k^{q}(x, a+(x-a) / 2) \mathrm{d} \nu\right)^{1 / q}(t-a)^{1 / p^{\prime}}<\infty .
$$

Moreover, there exist positive constants $b_{1}$ and $b_{2}$ depending only on $d_{1}, d_{2}, p$ and $q$ such that the inequality

$$
b_{1} B \leqslant\|K\| \leqslant b_{2} B
$$

is fulfilled. (If the constants $d_{1}$ and $d_{2}$ from Definitions 2.3 and 2.4 do not depend on a and $b$, then the constants $b_{1}, b_{2}$ are independent of $a$ and $b$.)

Proof. First we prove the theorem when $b=\infty$. Let $f \geqslant 0$. Then we have

$$
\begin{aligned}
\|K f\|_{L_{\nu}^{q}(a, \infty)} \leqslant\left(\int_{(a, \infty)}\left(\int_{a}^{a+(x-a) / 2} k(x, y) f(y) \mathrm{d} y\right)^{q} \mathrm{~d} \nu\right)^{1 / q} & \\
& +\left(\int_{(a, \infty)}\left(\int_{a+(x-a) / 2}^{x} k(x, y) f(y) \mathrm{d} y\right)^{q} \mathrm{~d} \nu\right)^{1 / q} \equiv I_{1}+I_{2} .
\end{aligned}
$$


If $a<y<a+(x-a) / 2$, then $k(x, y) \leqslant k(x, a+(x-a) / 2)$, and, consequently, using Lemma 2.1, we obtain

$$
\begin{aligned}
I_{1} & \leqslant c_{1}\left(\int_{(a, \infty)} k^{q}(x, a+(x-a) / 2)\left(\int_{a}^{x} f(y) \mathrm{d} y\right)^{q} \mathrm{~d} \nu\right)^{1 / q} \\
& \leqslant c_{2} B\|f\|_{L^{p}(a, \infty)} .
\end{aligned}
$$

Using Hölder's inequality, the condition $k \in V_{p}$ and the notation $s_{j} \equiv a+2^{j}$, we find that

$$
\begin{aligned}
I_{2}^{q} & \leqslant \int_{(a, \infty)}\left(\int_{a+(x-a) / 2}^{x}(f(y))^{p} \mathrm{~d} y\right)^{q / p}\left(\int_{a+(x-a) / 2}^{x} k^{p^{\prime}}(x, y) \mathrm{d} y\right)^{q / p^{\prime}} \mathrm{d} \nu \\
& \leqslant c_{3} \int_{(a, \infty)}\left(\int_{a+(x-a) / 2}^{x}(f(y))^{p} \mathrm{~d} y\right)^{q / p}(x-a)^{q / p^{\prime}} k^{q}(x, a+(x-a) / 2) \mathrm{d} \nu \\
& \leqslant c_{3} \sum_{j \in \mathbb{Z}} \int_{\left[s_{j}, s_{j+1}\right)}\left(\int_{a+(x-a) / 2}^{x}(f(y))^{p} \mathrm{~d} y\right)^{q / p}(x-a)^{q / p^{\prime}} k^{q}(x, a+(x-a) / 2) \mathrm{d} \nu \\
& \leqslant c_{3} \sum_{j \in \mathbb{Z}}\left(\int_{s_{j-1}}^{s_{j+1}}(f(y))^{p} \mathrm{~d} y\right)^{q / p} \int_{\left[s_{j}, s_{j+1}\right)}(x-a)^{q / p^{\prime}} k^{q}(x, a+(x-a) / 2) \mathrm{d} \nu \\
& \leqslant c_{4} B^{q} \sum_{j \in \mathbb{Z}}\left(\int_{s_{j-1}}^{s_{j+1}}(f(y))^{p} \mathrm{~d} y\right)^{q / p} \leqslant c_{5} B^{q}\|f\|_{L^{p}(a, \infty)}^{q} .
\end{aligned}
$$

Now we prove the necessity. First we show that from the boundedness of the operator $K$ the following condition can be obtained:

$$
\tilde{B} \equiv \sup _{j \in \mathbb{Z}}\left(\int_{\left[s_{j}, s_{j+1}\right)} k^{q}(x, a+(x-a) / 2)(x-a)^{q / p^{\prime}} \mathrm{d} \nu\right)^{1 / q}<\infty .
$$

Let $f_{j}(y)=\chi_{\left(a, s_{j+1}\right)}(y)$, where $j \in \mathbb{Z}$. Then we have that

$$
\begin{aligned}
\left\|K f_{j}\right\|_{L_{\nu}^{q}(a, \infty)} & \geqslant\left(\int_{\left[s_{j}, s_{j+1}\right)}\left(K f_{j}(x)\right)^{q} \mathrm{~d} \nu\right)^{1 / q} \\
& \geqslant\left(\int_{\left[s_{j}, s_{j+1}\right)}\left(\int_{a+(x-a) / 2}^{x} f_{j}(y) k(x, y) \mathrm{d} y\right)^{q} \mathrm{~d} \nu\right)^{1 / q} \\
& \geqslant c_{6}\left(\int_{\left[s_{j}, s_{j+1}\right)} k^{q}(x, a+(x-a) / 2)(x-a)^{q} \mathrm{~d} \nu\right)^{1 / q} .
\end{aligned}
$$

Consequently, using the boundedness of $K$, we obtain $\tilde{B}<\infty$. Now we show that $B \leqslant c_{7} \tilde{B}$. Denote

$$
\left(\int_{[t, \infty)} k^{q}(x, a+(x-a) / 2) \mathrm{d} \nu\right)^{1 / q}(t-a)^{1 / p^{\prime}} \equiv B(t) .
$$

Let $t \in(a, \infty)$; then $t \in\left[s_{m}, s_{m+1}\right)$ for some $m \in \mathbb{Z}$. 
We have

$$
\begin{aligned}
B^{q}(t) & \leqslant\left(\int_{\left[s_{m}, \infty\right)} k^{q}(x, a+(x-a) / 2) \mathrm{d} \nu\right) 2^{(m+1) q / p^{\prime}} \\
& =c_{8} 2^{m q / p^{\prime}} \sum_{j=m}^{+\infty} \int_{\left[s_{j}, s_{j+1}\right)} k^{q}(x, a+(x-a) / 2) \mathrm{d} \nu \\
& \leqslant c_{9} \tilde{B}^{q} 2^{m q / p^{\prime}} \sum_{j=m}^{+\infty} 2^{-j q / p^{\prime}}=c_{10} \tilde{B}
\end{aligned}
$$

where $c_{10}$ depends only on $q$ and $p$.

The case $b \leqslant \infty$ can be proved analogously. In this case we take $s_{j}=a+(b-a) 2^{j}$. (It is clear that $(a, b)=\cup_{j \leqslant 0}\left[s_{j-1}, s_{j}\right)$.)

Remark 3.2. There exist positive constants $a_{1}, a_{2}, a_{3}$ and $a_{4}$ depending only on $p$ and $q$ such that

$$
a_{1} B \leqslant \tilde{B} \leqslant a_{2} B
$$

if $b=\infty$, where $\tilde{B}$ is from $(3.1)$ and

$$
a_{3} B \leqslant \bar{B} \leqslant a_{4} B
$$

if $b<\infty$, where

$$
\bar{B}=\sup _{j \leqslant 0}\left(\int_{\left[a+(b-a) 2^{j-1}, a+(b-a) 2^{j}\right)} k^{q}(x, a+(x-b) / 2)(x-a)^{q / p^{\prime}} \mathrm{d} \nu\right)^{1 / q} .
$$

Indeed, let $b=\infty$. Then the inequality $a_{1} B \leqslant \tilde{B}$ follows from the proof of Theorem 3.1. Moreover,

$$
\begin{aligned}
\left(\int_{\left[a+2^{j}, a+2^{j+1}\right)} k^{q}(x, a+\right. & \left.(x-a) / 2)(x-a)^{q / p^{\prime}} \mathrm{d} \nu\right)^{1 / q} \\
& \leqslant c_{1}\left(\int_{\left[a+2^{j}, a+2^{j+1}\right)} k^{q}(x, a+(x-a) / 2) \mathrm{d} \nu\right)^{1 / q} 2^{j / p^{\prime}} \leqslant c_{1} B
\end{aligned}
$$

for every $j \in \mathbb{Z}$. Consequently, $\tilde{B} \leqslant a_{2} B$, where $a_{2}$ depends only on $p$ and $q$. We have an analogous result for $\bar{B}$.

Let $g$ be a $\nu$-measurable positive function on $(a, b)$ and let

$$
K^{\prime} g(y)=\int_{y}^{b} k(x, y) g(x) \mathrm{d} \nu
$$

where $y \in(a, b)$.

From the duality arguments we can derive the following result. 
Theorem 3.3. Let $-\infty<a<b \leqslant+\infty$ and let $1<p \leqslant q<\infty$. Suppose that $k \in V \cap V_{q^{\prime}}$. Then the operator $K^{\prime}$ is bounded from $L_{\nu}^{p}(a, b)$ to $L^{q}(a, b)$ if and only if

$$
B^{\prime}=\sup _{a<t<b}\left(\int_{[t, b)} k^{p^{\prime}}(x, a+(x-a) / 2) \mathrm{d} \nu\right)^{1 / p^{\prime}}(t-a)^{1 / q}<\infty .
$$

Moreover, there exist positive constants $b_{1}$ and $b_{2}$ depending only on $d_{1}, d_{2}, p$ and $q$ such that

$$
b_{1} B^{\prime} \leqslant\left\|K^{\prime}\right\| \leqslant b_{2} B^{\prime}
$$

Now we consider the case $q<p$. We shall assume that $v$ and $w$ are Lebesguemeasurable, a.e. positive functions on $(a, b)$.

Theorem 3.4. Let $-\infty<a<b \leqslant+\infty, 0<q<p<\infty$ and let $p>1$. Suppose that $k \in V \cap V_{p}$. Then the operator $K$ is bounded from $L^{p}(a, b)$ to $L_{v}^{q}(a, b)$ if and only if

$$
B_{1}=\left(\int_{a}^{b}\left(\int_{x}^{b} k^{q}(t, a+(t-a) / 2) v(t) \mathrm{d} t\right)^{p /(p-q)}(x-a)^{p(q-1) /(p-q)} \mathrm{d} x\right)^{(p-q) / p q}<\infty .
$$

Moreover, there exist positive constants $b_{1}$ and $b_{2}$ such that

$$
b_{1} B_{1} \leqslant\|K\| \leqslant b_{2} B_{1}
$$

Proof. We prove the theorem when $b=\infty$. The case $b<\infty$ can be proved similarly. Let $f \geqslant 0$. Then we have

$$
\begin{aligned}
\|K f\|_{L_{v}^{q}(a, \infty)}^{q} \leqslant c_{1} \int_{a}^{\infty}\left(\int_{a}^{a+(x-a) / 2} f(y) k(x, y) \mathrm{d} y\right)^{q} v(x) \mathrm{d} x & \\
& \quad+c_{1} \int_{a}^{\infty}\left(\int_{a+(x-a) / 2}^{x} f(y) k(x, y) \mathrm{d} y\right)^{q} v(x) \mathrm{d} x=\bar{I}_{1}+\bar{I}_{2} .
\end{aligned}
$$

Using Lemma 2.2, we obtain $\bar{I}_{1} \leqslant c_{2} B_{1}^{q}\|f\|_{L^{p}(a, \infty)}^{q}$, where $c_{2}$ depends only on $p, q$ and $d_{1}$. By Hölder's inequality and the condition $k \in V_{p}$ we find that

$$
\begin{aligned}
\bar{I}_{2} & \leqslant c_{3} \int_{a}^{\infty}\left(\int_{a+(x-a) / 2}^{x}(f(y))^{p} \mathrm{~d} y\right)^{q / p}(x-a)^{q / p^{\prime}} k^{q}(x, a+(x-a) / 2) v(x) \mathrm{d} x \\
& =c_{3} \sum_{j \in \mathbb{Z}} \int_{s_{j}}^{s_{j+1}}\left(\int_{a+(x-a) / 2}^{x}(f(y))^{p} \mathrm{~d} y\right)^{q / p}(x-a)^{q / p^{\prime}} k^{q}(x, a+(x-a) / 2) v(x) \mathrm{d} x \\
& \leqslant c_{3} \sum_{j \in \mathbb{Z}}\left(\int_{s_{j-1}}^{s_{j+1}}(f(y))^{p} \mathrm{~d} y\right)^{q / p} \int_{s_{j}}^{s_{j+1}}(x-a)^{q / p^{\prime}} k^{q}(x, a+(x-a) / 2) v(x) \mathrm{d} x,
\end{aligned}
$$


where $s_{j}=a+2^{j}$. Using Hölder's inequality again, we have

$$
\begin{aligned}
& \bar{I}_{2} \leqslant c_{3}\left(\sum_{j \in \mathbb{Z}} \int_{s_{j-1}}^{s_{j+1}}(f(y))^{p} \mathrm{~d} y\right)^{q / p} \\
& \times\left(\sum_{j \in \mathbb{Z}}\left(\int_{s_{j}}^{s_{j+1}}(x-a)^{q / p^{\prime}} k^{q}(x, a+(x-a) / 2) v(x) \mathrm{d} x\right)^{p /(p-q)}\right)^{(p-q) / p} \\
& \leqslant c_{4} \bar{B}_{1}^{q}\|f\|_{L^{p}(a, \infty)}^{q}
\end{aligned}
$$

where

$$
\bar{B}_{1} \equiv\left(\sum_{j \in \mathbb{Z}}\left(\int_{s_{j}}^{s_{j+1}}(x-a)^{q / p^{\prime}} k^{q}(x, a+(x-a) / 2) v(x) \mathrm{d} x\right)^{p /(p-q)}\right)^{(p-q) / p q} .
$$

Moreover,

$$
\begin{aligned}
& \bar{B}_{1}^{p q /(p-q)} \\
& \quad \leqslant c_{5} \sum_{j \in \mathbb{Z}} 2^{j q(p-1) /(p-q)}\left(\int_{s_{j}}^{s_{j}+1} k^{q}(x, a+(x-a) / 2) v(x) \mathrm{d} x\right)^{p /(p-q)} \\
& \quad \leqslant c_{5} \sum_{j \in \mathbb{Z}} \int_{s_{j-1}}^{s_{j}}(y-a)^{p(q-1) /(p-q)}\left(\int_{y}^{s_{j+1}} k^{q}(x, a+(x-a) / 2) v(x) \mathrm{d} x\right)^{p /(p-q)} \mathrm{d} y \\
& \quad \leqslant c_{5} \int_{a}^{\infty}(y-a)^{p(q-1) /(p-q)}\left(\int_{y}^{\infty} k^{q}(x, a+(x-a) / 2) v(x) \mathrm{d} x\right)^{p /(p-q)} \mathrm{d} y \\
& \quad=c_{5} B_{1}^{p q /(p-q)} .
\end{aligned}
$$

Consequently, $\bar{I}_{2} \leqslant c_{6} B_{1}^{q}\|f\|_{L^{p}(a, \infty)}^{q}$, where the positive constant $c_{6}$ depends only on $d_{2}$, $p$ and $q$.

Now we prove the necessity. Let the operator $K$ be bounded from $L^{p}(a, \infty)$ to $L_{v}^{q}(a, \infty)$. If we repeat the arguments used in the proof of Theorem 3.1, then we can obtain that, for every $x \in(a, \infty)$,

$$
\int_{x}^{\infty} v(t) k^{q}(t, a+(t-a) / 2) \mathrm{d} t<\infty
$$

Let $v_{n}(t)=v(t) \chi_{(a+1 / n, a+n)}(t)$, where $n$ is an integer with $n \geqslant 2$. Suppose that

$$
f_{n}(x)=\left(\int_{x}^{\infty} v_{n}(t) k^{q}(t, a+(t-a) / 2) \mathrm{d} t\right)^{1 /(p-q)}(x-a)^{(q-1) /(p-q)} .
$$


Then, by integration by parts we obtain

$$
\begin{aligned}
&\left\|f_{n}\right\|_{L^{p}(a, \infty)}=\left(\int_{a}^{\infty}\left(\int_{x}^{\infty} v_{n}(t) k^{q}(t, a+(t-a) / 2) \mathrm{d} t\right)^{p /(p-q)}(x-a)^{(q-1) p /(p-q)} \mathrm{d} x\right)^{1 / p} \\
&=c_{7}\left(\int_{a}^{\infty}\left(\int_{x}^{\infty} v_{n}(t) k^{q}(t, a+(t-a) / 2) \mathrm{d} t\right)^{q /(p-q)}\right. \\
& \\
&\left.\quad \times(x-a)^{(p-1) q /(p-q)} v_{n}(x) k^{q}(x, a+(x-a) / 2) \mathrm{d} x\right)^{1 / p}<\infty .
\end{aligned}
$$

On the other hand,

$$
\begin{aligned}
\left\|K f_{n}\right\|_{L_{v}^{q}(a, \infty)} & \\
\geqslant & c_{8}\left(\int_{a}^{\infty} v(x)\left(\int_{a+(x-a) / 2}^{x} f_{n}(t) k(x, t) \mathrm{d} t\right)^{q} \mathrm{~d} x\right)^{1 / q} \\
\geqslant & c_{9}\left(\int_{a}^{\infty} v_{n}(x) k^{q}(x, a+(x-a) / 2)\left(\int_{x}^{\infty} v_{n}(t) k^{q}(x, a+(t-a) / 2) \mathrm{d} t\right)^{q /(p-q)}\right. \\
& \left.\times\left(\int_{a+(x-a) / 2}^{x}(t-a)^{(q-1) /(p-q)} \mathrm{d} t\right)^{q} \mathrm{~d} x\right)^{1 / q} \\
\geqslant & c_{10}\left(\int_{a}^{\infty} v_{n}(x) k^{q}(x, a+(x-a) / 2)\right. \\
& \left.\times\left(\int_{x}^{\infty} v_{n}(t) k^{q}(t, a+(t-a) / 2) \mathrm{d} t\right)^{q /(p-q)}(x-a)^{(p-1) q /(p-q)} \mathrm{d} x\right)^{1 / q} \\
= & c_{11}\left(\int_{a}^{\infty}\left(\int_{x}^{\infty} v_{n}(t) k^{q}(t, a+(t-a) / 2) \mathrm{d} t\right)^{p /(p-q)}(x-a)^{p(q-1) /(p-q)} \mathrm{d} x\right)^{1 / q} .
\end{aligned}
$$

From the boundedness of the operator $K$ we get

$$
\left(\int_{a}^{\infty}\left(\int_{x}^{\infty} k^{q}(t, a+(t-a) / 2) v_{n}(t) \mathrm{d} t\right)^{p /(p-q)}(x-a)^{p(q-1) /(p-q)} \mathrm{d} x\right)^{(p-q) / p q} \leqslant c,
$$

where the positive constant $c$ does not depend on $n$. By Fatou's Lemma we finally obtain $B_{1}<\infty$.

Now let

$$
\tilde{K} f(x)=\int_{x}^{b} f(y) k(y, x) w(y) \mathrm{d} y,
$$

where $w$ is a Lebesgue-measurable a.e. positive function on $(a, b)$. From the duality arguments and from Theorem 3.4 we obtain the following theorem.

Theorem 3.5. Let $-\infty<a<b \leqslant+\infty$ and let $1<q<p<\infty$. Suppose that $k \in V \cap V_{q^{\prime}}$. Then the operator $\tilde{K}$ is bounded from $L_{w}^{p}(a, b)$ to $L^{q}(a, b)$ if and only if

$$
\tilde{B}_{1}=\left(\int_{a}^{b}\left(\int_{x}^{b} k^{p^{\prime}}(t,(t-a) / 2) w(t) \mathrm{d} t\right)^{q(p-1) /(p-q)}(x-a)^{q /(p-q)} \mathrm{d} x\right)^{(p-q) / p q}<\infty .
$$


Moreover, there exist positive constants $\tilde{b}_{1}$ and $\tilde{b}_{2}$ such that

$$
\tilde{b}_{1} \tilde{B}_{1} \leqslant\|\tilde{K}\| \leqslant \tilde{b}_{2} \tilde{B}_{1} \text {. }
$$

\section{The compactness criteria}

In this section we investigate the compactness of the operators $K$ and $K^{\prime}$. The following theorem is true.

Theorem 4.1. Let $-\infty<a<b<+\infty, 1<p \leqslant q<\infty$ and let $k \in V \cap V_{p}$. Suppose that $\nu$ is a separable measure (i.e. $L_{\nu}^{q}(a, b)$ is a separable space). Then the following statements are equivalent:

(i) the operator $K$ is compact from $L^{p}(a, b)$ to $L_{\nu}^{q}(a, b)$;

(ii) $B<\infty$ and $\lim _{c \rightarrow a+} B_{c}=0$, where

$$
B_{c} \equiv \sup _{a<t<c}\left(\int_{[t, c)} k^{q}(x, a+(x-a) / 2) \mathrm{d} \nu\right)^{1 / q}(t-a)^{1 / p^{\prime}}
$$

(iii) $\bar{B}<\infty$ and $\lim _{j \rightarrow-\infty} \bar{B}(j)=0$, where

$$
\bar{B}(j)=\left(\int_{\left[s_{j-1}, s_{j}\right)} k^{q}(x, a+(x-a) / 2)(x-a)^{q / p^{\prime}} \mathrm{d} \nu\right)^{1 / q}
$$

and $s_{j}=a+(b-a) 2^{j}$.

Proof. First we prove that (ii) implies (i). Let $c \in(a, b)$ and represent $K$ as follows:

$$
K=\chi_{(a, c)} K+\chi_{[c, b)} K=P_{1 c}+P_{2 c} .
$$

For $P_{2 c}$ we have

$$
P_{2 c} f(x)=\chi_{[c, b)}(x) \int_{a}^{b} T_{1}(x, y) \mathrm{d} y,
$$

where $T_{1}(x, y)=k(x, y)$ when $a<y<x<b$ and $T_{1}(x, y)=0$ if $a<x \leqslant y<b$. Consequently,

$$
\begin{aligned}
S \equiv & \int_{[c, b)}\left(\int_{a}^{b}\left(T_{1}(x, y)\right)^{p^{\prime}} \mathrm{d} y\right)^{q / p^{\prime}} \mathrm{d} \nu \\
= & \int_{[c, b)}\left(\int_{a}^{x}(k(x, y))^{p^{\prime}} \mathrm{d} y\right)^{q / p^{\prime}} \mathrm{d} \nu \\
\leqslant & c_{1} \int_{[c, b)}\left(\int_{a}^{a+(x-a) / 2}(k(x, y))^{p^{\prime}} \mathrm{d} y\right)^{q / p^{\prime}} \mathrm{d} \nu \\
& \quad+c_{1} \int_{[c, b)}\left(\int_{a+(x-a) / 2}^{x}(k(x, y))^{p^{\prime}} \mathrm{d} y\right)^{q / p^{\prime}} \mathrm{d} \nu \\
\equiv & S_{1}+S_{2} .
\end{aligned}
$$


If $a<y<a+(x-a) / 2$, then $k(x, y) \leqslant d_{1} k(x, a+(x-a) / 2)$ and therefore we have

$$
\begin{aligned}
S_{1} & \leqslant c_{2} \int_{[c, b)} k^{q}(x, a+(x-a) / 2)((x-a) / 2)^{q / p^{\prime}} \mathrm{d} \nu \\
& \leqslant c_{2}\left(\int_{[c, b)} k^{q}(x, a+(x-a) / 2) \mathrm{d} \nu\right)((b-a) / 2)^{q / p^{\prime}}<\infty .
\end{aligned}
$$

Using the condition $k \in V_{p}$, for $S_{2}$ we obtain

$$
S_{2} \leqslant c_{3} \int_{[c, b)} k^{q}(x, a+(x-a) / 2)(x-a)^{q / p^{\prime}} \mathrm{d} \nu<\infty .
$$

Finally, we have $S<\infty$ and, by Theorem A, we conclude that $P_{2 c}$ is compact. Moreover, by virtue of Theorem 3.1 we have $\left\|P_{1 c}\right\| \leqslant c_{4} B_{c}$, where the positive constant $c_{4}$ does not depend on $c$. Consequently,

$$
\left\|K-P_{2 c}\right\| \leqslant c_{4} B_{c} \rightarrow 0
$$

as $c \rightarrow a$ and the operator $K$ is compact as a limit of compact operators. Now we prove that (i) implies (iii). Let $j \in \mathbb{Z}, j \leqslant 0$ and let

$$
f_{j}(y)=\chi_{\left(a, a+(b-a) 2^{j}\right)}(y)\left((b-a) 2^{j}\right)^{-1 / p} .
$$

Then for $\varphi \in L^{p^{\prime}}(a, b)$ we have

$$
\begin{aligned}
\left|\int_{a}^{b} f_{j}(y) \varphi(y) \mathrm{d} y\right| & \leqslant\left(\int_{a}^{s_{j}}\left|f_{j}(y)\right|^{p} \mathrm{~d} y\right)^{1 / p}\left(\int_{a}^{s_{j}}|\varphi(y)|^{p^{\prime}} \mathrm{d} y\right)^{1 / p^{\prime}} \\
& =\left(\int_{a}^{s_{j}}|\varphi(y)|^{p^{\prime}} \mathrm{d} y\right)^{1 / p^{\prime}} \rightarrow 0
\end{aligned}
$$

as $j \rightarrow-\infty$ (here $\left.s_{j}=a+(b-a) 2^{j}\right)$. On the other hand,

$$
\begin{aligned}
\left\|K f_{j}\right\|_{L_{\nu}^{q}(a, b)} & \geqslant\left(\int_{\left[s_{j-1}, s_{j}\right)}\left(K f_{j}(x)\right)^{q} \mathrm{~d} \nu\right)^{1 / q} \\
& \geqslant\left(\int_{\left[s_{j-1}, s_{j}\right)} k^{q}(x, a+(x-a) / 2)\left(\int_{a+(x-a) / 2}^{x} f_{j}(y) \mathrm{d} y\right)^{q} \mathrm{~d} \nu\right)^{1 / q} \\
& \geqslant c_{5}\left(\int_{\left[s_{j-1}, s_{j}\right)} k^{q}(x, a+(x-a) / 2)(x-a)^{q} \mathrm{~d} \nu\right)^{1 / q}\left((b-a) 2^{j}\right)^{-1 / p} \\
& \geqslant c_{6} \bar{B}(j) .
\end{aligned}
$$

As a compact operator maps a weakly convergent sequence into a strongly convergent form, we have that $\lim _{j \rightarrow-\infty} \bar{B}(j)=0$. The fact that $\bar{B}<\infty$ follows from Remark 3.2 and Theorem 3.1.

Now we prove that (ii) follows from (iii). Let $c \in(a, b)$. Then there exists an integer $m$ with $m \leqslant 0$ such that $c \in\left[s_{m-1}, s_{m}\right)$. We have

$$
B_{c} \leqslant \sup _{a<t<s_{m}}\left(\int_{\left[t, s_{m}\right)} k^{q}(x, a+(x-a) / 2) \mathrm{d} \nu\right)^{1 / q}(t-a)^{1 / p^{\prime}}=B_{s_{m}} .
$$


Denote

$$
B_{s_{m}}(t) \equiv\left(\int_{\left[t, s_{m}\right)} k^{q}(x, a+(x-a) / 2) \mathrm{d} \nu\right)^{1 / q}(t-a)^{1 / p^{\prime}}
$$

Let $t \in\left(a, s_{m}\right)$, then $t \in\left[s_{n-1}, s_{n}\right)$ for some integer $n \leqslant m$. We obtain

$$
\begin{aligned}
B_{s_{m}}^{q}(t) & \leqslant\left(\int_{\left[s_{n-1}, s_{m}\right)} k^{q}(x, a+(x-a) / 2) \mathrm{d} \nu\right)\left[(b-a) 2^{n}\right]^{q / p^{\prime}} \\
& =\left[(b-a) 2^{n}\right]^{q / p^{\prime}} \sum_{j=n}^{m} \int_{\left[s_{j-1}, s_{j}\right)} k^{q}(x, a+(x-a) / 2) \mathrm{d} \nu \\
& \leqslant c_{7}\left[(b-a) 2^{n}\right]^{q / p^{\prime}} \sum_{j=n}^{m}\left[(b-a) 2^{j}\right]^{-q / p^{\prime}} \\
& \times \int_{\left[s_{j-1}, s_{j}\right)} k^{q}(x, a+(x-a) / 2)(x-a)^{q / p^{\prime}} \mathrm{d} \nu \\
& \leqslant c_{7}\left(\sup _{j \leqslant m} \bar{B}(j)\right)^{q}\left[(b-a) 2^{n}\right]^{q / p^{\prime}} \sum_{j=n}^{m}\left[(b-a) 2^{j}\right]^{-q / p^{\prime}} \\
& \leqslant c_{8}\left(\sup _{j \leqslant m} \bar{B}(j)\right)^{q} \equiv c_{8} \bar{B}_{m}^{q} .
\end{aligned}
$$

Consequently,

$$
B_{s_{m}} \leqslant c_{9} \bar{B}_{m}
$$

If $c \rightarrow a$, then $s_{m} \rightarrow a$. Therefore $\bar{B}_{m} \rightarrow 0$ as $\lim _{j \rightarrow-\infty} \bar{B}(j)=0$. Finally, we get $\lim _{c \rightarrow a+} B_{c}=0$. The condition $B<\infty$ follows from Remark 3.2. So we conclude that (ii) $\Longrightarrow$ (i) $\Longrightarrow$ (iii) $\Longrightarrow$ (ii).

From the duality argument we obtain the following theorem.

Theorem 4.2. Let $-\infty<a<b<+\infty$ and let $1<p \leqslant q<\infty$. Suppose that $\nu$ is a separable measure (i.e. $L_{\nu}^{p^{\prime}}(a, b)$ is separable) and $k \in V \cap V_{q^{\prime}}$. Then the following statements are equivalent:

(i) the operator $K^{\prime}$ is compact from $L_{\nu}^{p}(a, b)$ to $L^{q}(a, b)$;

(ii) $B^{\prime}<\infty$ and $\lim _{c \rightarrow a+} B_{c}^{\prime}=0$, where

$$
B_{c}^{\prime}=\sup _{a<t<c}\left(\int_{[t, c)} k^{p^{\prime}}(x, a+(x-a) / 2) \mathrm{d} \nu\right)^{1 / p^{\prime}}(t-a)^{1 / q}
$$

(iii)

$$
\bar{B}^{\prime} \equiv \sup _{j \leqslant 0}\left(\int_{\left[s_{j-1}, s_{j}\right)} k^{p^{\prime}}(x, a+(x-a) / 2)(x-a)^{p^{\prime} / q} \mathrm{~d} \nu\right)^{1 / p^{\prime}}<\infty
$$


and $\lim _{j \rightarrow-\infty} \bar{B}^{\prime}(j)=0$, where

$$
\bar{B}^{\prime}(j)=\left(\int_{\left[s_{j-1}, s_{j}\right)} k^{p^{\prime}}(x, a+(x-a) / 2)(x-a)^{p^{\prime} / q} \mathrm{~d} \nu\right)^{1 / p^{\prime}}
$$

and

$$
s_{j}=a+(b-a) 2^{j} .
$$

Theorem 4.3. Let $-\infty<a<b \leqslant+\infty, 0<q<p<\infty$ and let $p>1$. Suppose that $k \in V \cap V_{p}$. Then the operator $K$ is compact from $L^{p}(a, b)$ to $L_{v}^{q}(a, b)$ if and only if $B_{1}<\infty$.

Proof. The sufficiency of the theorem can be derived in the same way as in the proof of Theorem 4.1. (It also follows from the well-known Ando's Theorem [1] .) Theorem 3.4 implies the necessity.

The following theorem can be derived from Theorem 4.3.

Theorem 4.4. Let $-\infty<a<b \leqslant+\infty$ and let $1<q<p<\infty$. Suppose that $k \in V \cap V_{q^{\prime}}$. Then the operator $\tilde{K}$ is compact from $L_{w}^{p}(a, b)$ to $L^{q}(a, b)$ if and only if $\tilde{B}_{1}<\infty$.

\section{The measure of non-compactness}

In the non-compact case it is useful to estimate the distance of the operator $K$ from the space of compact operators.

Let $X$ and $Y$ be Banach function spaces. Denote by $\mathcal{B}(X, Y)$ the space of all linear bounded operators from $X$ to $Y$. Let $\mathcal{K}(X, Y)$ be a class of all linear compact operators from $X$ to $Y$. Suppose that $\mathcal{F}_{r}(X, Y)$ is a space of operators with finite rank.

We shall assume that $v$ is a Lebesgue-measurable a.e. positive function on $(a, b)$, where $-\infty<a<b \leqslant+\infty$.

The following lemma is true (see [16] and [3, Corollary V.5.4]).

Lemma 5.1. Let $1 \leqslant p<\infty,-\infty<a<b \leqslant+\infty$ and let $P \in \mathcal{B}(X, Y)$, where $Y=L_{v}^{p}(a, b)$. Then

$$
\operatorname{dist}(P, \mathcal{K}(X, Y))=\operatorname{dist}\left(P, \mathcal{F}_{r}(X, Y)\right) .
$$

We also need the following lemma (see [16] and [3, Lemma V.5.6]).

Lemma 5.2. Let $1 \leqslant p<\infty,-\infty<a<b \leqslant+\infty$ and let $Y=L_{v}^{p}(a, b)$. Suppose that $P \in \mathcal{F}_{r}(X, Y)$ and $\epsilon>0$. Then there exist $T \in \mathcal{F}_{r}(X, Y)$ and $[\alpha, \beta] \subset(a, b)$ such that

$$
\|P-T\|<\epsilon
$$

and

$$
\operatorname{supp} T f \subset[\alpha, \beta]
$$

for every $f \in X$. 
Theorem 5.3. Let $1<p \leqslant q<\infty,-\infty<a<b<+\infty$ and let $k \in V \cap V_{p}$. Suppose that $K$ is bounded from $X$ to $Y$, where $X=L^{p}(a, b)$ and $Y=L_{v}^{q}(a, b)$. Then the inequality

$$
b_{1} J \leqslant \operatorname{dist}(K, \mathcal{K}(X, Y)) \leqslant b_{2} J
$$

is fulfilled, where the positive constants $b_{1}$ and $b_{2}$ depend only on $p, q, d_{1}$ and $d_{2}$, $J=\lim _{c \rightarrow a+} R_{c}$ and

$$
R_{c}=\sup _{a<t<c}\left(\int_{t}^{c} k^{q}(x, a+(x-a) / 2) v(x) \mathrm{d} x\right)^{1 / q}(t-a)^{1 / p^{\prime}}
$$

( $d_{1}$ and $d_{2}$ are from Definitions 2.3 and 2.4).

Proof. As we know from the proof of Theorem 4.1,

$$
\left\|K-\bar{P}_{c}\right\| \leqslant c_{1} R_{c},
$$

where $\bar{P}_{c}$ is a compact operator for every $c$. From the last inequality we can obtain

$$
\operatorname{dist}(K, \mathcal{K}(X, Y)) \leqslant c_{1} J
$$

where $c_{1}$ depends only on $p, q, d_{1}$ and $d_{2}$. Now we show that

$$
\operatorname{dist}(K, \mathcal{K}(X, Y)) \geqslant b_{1} J
$$

Let $\lambda>\operatorname{dist}(K, \mathcal{K}(X, Y))$. Then by Lemma 5.1 there exists $P \in \mathcal{F}_{r}(X, Y)$ such that $\|K-P\|<\lambda$. On the other hand, using Lemma 5.2, for $\epsilon=(\lambda-\|K-P\|) / 2$ there exist $T \in \mathcal{F}_{r}(X, Y)$ and $[\alpha, \beta] \subset(a, b)$ such that

$$
\|P-T\|<\epsilon
$$

and

$$
\operatorname{supp} T f \subset[\alpha, \beta]
$$

From (5.3) we obtain

$$
\|K f-T f\|_{Y} \leqslant \lambda\|f\|_{X}
$$

for every $f \in X$. Consequently, we have

$$
\int_{a}^{\alpha}|K f(x)|^{q} v(x) \mathrm{d} x+\int_{\beta}^{b}|K f(x)|^{q} v(x) \mathrm{d} x \leqslant \lambda^{q}\|f\|_{X}^{q}
$$

for every $f \in X$.

Let us choose $n \in \mathbb{Z}$ such that $a+(b-a) 2^{n}<\alpha$. Assume that $j \in \mathbb{Z}, j \leqslant n$ and $f_{j}(y)=\chi_{\left(a, s_{j}\right)}(y)$, where $s_{j}=a+(b-a) 2^{j}$. Then we obtain

$$
\begin{aligned}
\int_{s_{j-1}}^{s_{j}}\left|K f_{j}(x)\right|^{q} v(x) \mathrm{d} x & \geqslant \int_{s_{j-1}}^{s_{j}}\left(\int_{a+(x-a) / 2}^{x} k(x, y) f(y) \mathrm{d} y\right)^{q} v(x) \mathrm{d} x \\
& \geqslant c_{2} \int_{s_{j-1}}^{s_{j}} k^{q}(x, a+(x-a) / 2)(x-a)^{q} v(x) \mathrm{d} x .
\end{aligned}
$$


On the other hand,

$$
\left\|f_{j}\right\|_{X}^{q}=\left((b-a) 2^{j}\right)^{q / p}
$$

and by (5.5) we find

$$
c_{3} R(j) \equiv c_{3}\left(\int_{s_{j-1}}^{s_{j}} k^{q}(x, a+(x-a) / 2)(x-a)^{q / p^{\prime}} v(x) \mathrm{d} x\right)^{1 / q} \leqslant \lambda
$$

for every integer $j, j \leqslant n$. Consequently, $\sup _{j \leqslant n} R(j) \leqslant c_{4} \lambda$ for every integer $n$ with the condition $a+(b-a) 2^{n}<\alpha$. Therefore we have

$$
\lim _{n \rightarrow-\infty} \sup _{j \leqslant n} R(j) \leqslant c_{4} \lambda .
$$

Let $c \in(a, \alpha)$; then $c \in\left[s_{m-1}, s_{m}\right)$ for some $m=m(c), m \in \mathbb{Z}$. We obtain (see the proof of Theorem 4.1)

$$
R_{c} \leqslant c_{5} \sup _{n \leqslant m} R(n) \equiv c_{5} \bar{R}_{m}
$$

From the last inequality we have

$$
\lim _{c \rightarrow a+} R_{c} \leqslant c_{5} \lim _{m \rightarrow-\infty} \bar{R}_{m} \leqslant c_{6} \lambda,
$$

where $c_{6}$ does not depend on $a$ and $b$. Finally, we obtain inequality (5.2) and consequently (5.1) is fulfilled.

Now we give the estimate of measure of non-compactness for the Riemann-Liouville operator $R_{\alpha}$. The following theorem is true.

Theorem 5.4. Let $1<p \leqslant q<\infty$ and let $\alpha>1 / p$. Suppose that $R_{\alpha}$ is bounded from $X$ to $Y$, where $X=L^{p}(0, \infty), Y=L_{v}^{q}(0, \infty)$. Then the inequality

$$
b_{1} I \leqslant \operatorname{dist}\left(R_{\alpha}, \mathcal{K}(X, Y)\right) \leqslant b_{2} I
$$

holds, where $I=\lim _{c \rightarrow 0} I_{c}+\lim _{d \rightarrow \infty} I_{d}$,

$$
\begin{aligned}
I_{c} & =\sup _{0<t<c}\left(\int_{t}^{c} \frac{v(x)}{x^{(1-\alpha) q}} \mathrm{~d} x\right)^{1 / q} t^{1 / p^{\prime}}, \\
I_{d} & =\sup _{t>d}\left(\int_{t}^{\infty} \frac{v(x)}{x^{(1-\alpha) q}} \mathrm{~d} x\right)^{1 / q}(t-d)^{1 / p^{\prime}},
\end{aligned}
$$

and the positive constants $b_{1}$ and $b_{2}$ depend only on $p, q$ and $\alpha$.

Proof. If we repeat the arguments used in the proof of Theorem 5 in $[\mathbf{1 3}]$, then we can obtain

$$
\operatorname{dist}\left(R_{\alpha}, \mathcal{K}(X, Y)\right) \leqslant b_{2} I
$$


Now let $\lambda>\operatorname{dist}\left(R_{\alpha}, \mathcal{K}(X, Y)\right)$. Then by Lemma 5.1 there exists $P \in \mathcal{F}_{r}(X, Y)$ such that $\left\|R_{\alpha}-P\right\|<\lambda$. By virtue of Lemma 5.2 for $\epsilon=\left(\lambda-\left\|R_{\alpha}-P\right\|\right) / 2$ there are $T \in \mathcal{F}_{r}(X, Y)$ and $[\alpha, \beta] \subset(0, \infty)$ such that (5.3) and (5.4) hold. From (5.3) we obtain

$$
\left\|R_{\alpha} f-T f\right\|_{Y} \leqslant \lambda\|f\|_{X}
$$

for every $f \in X$. Further, from (5.3), (5.4) and (5.6) we can obtain

$$
\int_{0}^{\alpha}\left|R_{\alpha} f(x)\right|{ }^{q} v(x) \mathrm{d} x+\int_{\beta}^{\infty}\left|R_{\alpha} f(x)\right|{ }^{q} v(x) \mathrm{d} x \leqslant \lambda^{q}\|f\|_{L^{p}(0, \infty)}^{q} .
$$

Let $d \geqslant \beta$ and let $t \in(d, \infty)$. Then for $f_{t}(y)=\chi_{(d / 2, t / 2)}(y)$ we have

$$
\begin{aligned}
\int_{t}^{\infty}\left|R_{\alpha} f_{t}(x)\right|^{q} v(x) \mathrm{d} x & \geqslant \int_{t}^{\infty}\left(\int_{d / 2}^{t / 2} \frac{f_{t}(y)}{(x-y)^{1-\alpha}} \mathrm{d} y\right)^{q} v(x) \mathrm{d} x \\
& \geqslant c_{1}\left(\int_{t}^{\infty} x^{(\alpha-1) q} v(x) \mathrm{d} x\right)(t-d)^{q}
\end{aligned}
$$

On the other hand,

$$
\|f\|_{L^{p}(0, \infty)}^{q}=c_{2}(t-d)^{q / p}
$$

whence

$$
\lambda \geqslant c_{3}\left(\int_{t}^{\infty} x^{(\alpha-1) q} v(x) \mathrm{d} x\right)^{1 / q}(t-d)^{1 / p^{\prime}}
$$

for $t>d$. Consequently, $\lambda \geqslant c_{3} I_{d}$ for every $d, d>\beta$. From the last inequality we have

$$
c_{3} \lim _{d \rightarrow \infty} I_{d} \leqslant \lambda \text {. }
$$

As $\lambda$ is an arbitrary number greater than $\operatorname{dist}\left(R_{\alpha}, \mathcal{K}(X, Y)\right)$, we conclude that

$$
c_{3} \lim _{d \rightarrow \infty} I_{d} \leqslant \operatorname{dist}\left(R_{\alpha}, \mathcal{K}(X, Y)\right) .
$$

Analogously we can show that

$$
c_{4} \lim _{c \rightarrow 0} I_{c} \leqslant \operatorname{dist}\left(R_{\alpha}, \mathcal{K}(X, Y)\right) .
$$

Consequently,

$$
b_{1} I \leqslant \operatorname{dist}\left(R_{\alpha}, \mathcal{K}(X, Y)\right) .
$$

An analogous theorem with two weights for the Hardy operator is proved in [5], while the similar problem for the Riemann-Liouville transforms $R_{\alpha}$ with $\alpha>1$ and for more general operators was solved in $[\mathbf{4}],[\mathbf{1 6}]$.

Acknowledgements. This work was supported by the Georgian Academy of Sciences grant no. 1.7. The author expresses his gratitude to Professor V. Kokilashvili for his interest in the present paper and to the referee for helpful remarks. 


\section{References}

1. T. ANDO, On compactness of integral operators, Indag. Math. (New Series) 24 (1962), $235-239$.

2. J. S. BRAdley, Hardy inequality with mixed norms, Can. Math. Bull. 21 (1978), 405-408.

3. D. E. Edmunds AND W. D. Evans, Spectral theory and differential operators (Oxford University Press, 1987).

4. D. E. Edmunds and V. D. Stepanov, The measure of noncompactness and approximation numbers of certain Volterra integral operators, Math. Annln 298 (1994), 41-66.

5. D. E. Edmunds, W. D. Evans And D. J. HARRIs, Approximation numbers of certain Volterra integral operators, J. Lond. Math. Soc. 37 (1988), 471-489.

6. I. Genebashvili, A. Gogatishvili and V. Kokilashvili, Solution of two-weight problems for integral transforms with positive kernels, Georgian Math. J. 3 (1996), 319-342.

7. I. Genebashvili, A. Gogatishvili, V. Kokilashvili and M. Krbec, Weight theory for integral transforms on spaces of homogeneous type, Pitman Monographs and Surveys in Pure and Applied Mathematics, no. 92 (Longman, Harlow, 1998).

8. L. P. Kantorovich And G. P. Akilov, Functional analysis (Pergamon, Oxford, 1982).

9. V. M. Kokilashvili, On Hardy's inequalities in weighted spaces (in Russian), Soobshch. Akad. Nauk Gruzin. SSR 96 (1979), 37-40.

10. V. Kokilashvili And A. Meskhi, Criteria for the boundedness and compactness for integral transforms with power-logarithmic kernels, Analysis Math. 27 (2001), in press.

11. J. F. MARTIN-REYES AND E. SAWYER, Weighted inequalities for Riemann-Liouville fractional integrals of order one and greater, Proc. Am. Math. Soc. 106 (1989), 727-733.

12. V. G. MAZ'YA, Sobolev spaces (Springer, Berlin, 1985).

13. A. Meskhi, Solution of some weight problems for the Riemann-Liouville and Weyl operators, Georgian Math. J. 5 (1998), 565-574.

14. A. Meskhi, Boundedness and compactness weighted criteria for Riemann-Liouville and one-sided maximal operators, Proc. A. Razmadze Math. Inst. 117 (1998), 126-128.

15. J. NEWman AND M. SolOMYAK, Two-sided estimates on singular values for a class of integral operators on the semi-axis, Integ. Eqns Operator Theory 20 (1994), 335-349.

16. B. OpIC, On the distance of the Riemann-Liouville operators from compact operators, Proc. Am. Math. Soc. 122 (1994), 495-501.

17. S. G. SAmko, A. A. Kilbas AND O. I. MARIChev, Integrals and derivatives. Theory and applications (Gordon and Breach, London, 1993).

18. G. Sinnamon And V. D. Stepanov, The weighted Hardy inequality: new proofs and the case $p=1$, J. Lond. Math. Soc. 54 (1996), 89-101.

19. V. D. Stepanov, Two-weighted estimates for Riemann-Liouville integrals, Report no. 39 (Mathematical Institute, Czechoslovak Academy of Science, 1988). 